Pacific Journal of Mathematics

THE MAZUR PROPERTY FOR COMPACT SETS 


\title{
THE MAZUR PROPERTY FOR COMPACT SETS
}

\author{
Abderrazzak Sersouri
}

\begin{abstract}
We give a "convex" characterization to the following smoothness property, denoted by $(C I)$ : every compact convex set is the intersection of balls containing it. This characterization is used to give a transfer theorem for property $(C I)$. As an application we prove that the family of spaces which have an equivalent norm with property $(C I)$ is stable under $c_{0}$ and $l_{p}$ sums for $1 \leq p<\infty$. We also prove that if $X$ has a transfinite Schauder basis, and $Y$ has an equivalent norm with property $(C I)$ then the space $X \hat{\otimes}_{\rho} Y$ has an equivalent norm with property $(C I)$, for every tensor norm $\rho$.

Similar results are obtained for the usual Mazur property $(I)$, that is, the family of spaces which have an equivalent norm with property $(I)$ is stable under $c_{0}$ and $l_{p}$ sums for $1<p<\infty$.
\end{abstract}

Introduction. Mazur [6] was the first who considered the following separation property, denoted by $(I)$ :

Every bounded closed convex set is the intersection of balls containing it.

Later, Phelps [7] proved that property $(I)$ is weaker than the Fréchet differentiability of the norm, and gave a dual characterization for $(I)$ in the finite dimensional case.

Phelps' theorem was extended to the infinite dimensional case in [3], where the property $(I)$ was dually characterized.

Here we will give another extension of Phelps' theorem by characterizing the following property, denoted by $(C I)$ :

Every compact convex set is an intersection of balls.

This property was recently introduced by Whitfield and Zizler [9].

We use this characterization to give a "transfer theorem" for property $(C I)$, which is analogous to the one given for property $(I)$ [2].

We also prove a stability result for property $(C I)$, which is of the same nature as the one given by Zizler for l.u.c. renormings [10]. Our proof can be modified to give a similar stability result for property (I).

Some renorming results of Whitfield-Zizler [9], and Deville [2] are particular cases of these stability results. 
Notation. Our notation is standard. A point $x \in X$ is said to be extremal if $x=0$ or $x /\|x\|$ is an extreme point of the unit ball of $X$. Similar conventions will be used for $w^{*}$-exposed points, $w^{*}$-denting points, and $w^{*}$-strongly exposed points.

The unit ball and the unit sphere of a Banach space $X$ will be denoted by $B(X)$ and $S(X)$ respectively. We also denote by $B(z, r)$ [resp. $S(z, r)$ ] the ball [resp. the sphere] centered at $z$ and of radius $r$ (the underlying Banach space is understood).

For a subset $C$ of a Banach space $X$ we denote by $c v(C)$ [resp. $\overline{c v}(C)$ ] the convex [resp. closed convex] hull of $C$.

1. Dual characterization for property $(C I)$. The following theorem is analogous to the one given for property $(I)$ [3]. Techniques used in the proof can be found in Phelps' paper [7].

Theorem 1. Let $X$ be a Banach space. The following properties are equivalent:

(i) Every compact convex set is the intersection of balls containing it.

(ii) The cone of extreme points of $X^{*}$ is dense in $X^{*}$ for the topology $\mathscr{T}$ of uniform convergence on compact sets of $X$.

Proof. (i) $\Rightarrow$ (ii). Let $f \in S\left(X^{*}\right), K$ a compact subset of $B(X)$, and $\varepsilon>0$. We want to find $g \in \operatorname{Ext}\left(B\left(X^{*}\right)\right)$, and $\lambda>0$, such that

$$
\|f-\lambda g\|_{K}=\operatorname{Sup}_{K}|f-\lambda g| \leq \varepsilon .
$$

Without loss of generality we can suppose that $K$ is absolutely convex and $\|f\|_{K} \geq 1-\varepsilon / 2$.

(Indeed, let $x \in B(X)$ such that $f(x)>1-\varepsilon / 2$, and let $L$ be the closed convex symmetric hull of $K \cup\{x\}$. The above mentioned reduction is then possible since $\|\cdot\|_{L} \geq\|\cdot\|_{K}$.) Let $u \in K$ be such that $f(u)=1-\varepsilon / 2$, and put $u^{\prime}=(\varepsilon / 4) u$, and $D=K \cap f^{-1}(0)$. By (i), there exists $z \in X, r>0$, such that $u^{\prime} \notin B(z, r)$, and $D \subset B(z, r)$.

Let $w$ be the unique element of $\left[S(z, r) \cap c v\left(u^{\prime}, z\right)\right]$. Put $x=$ $(w-z) / r$, and let $g \in \operatorname{Ext}\left(B\left(X^{*}\right)\right)$ such that $\|x\|=g(x)=1$. Then it is easy to see that:

$$
0 \leq g(w)=\operatorname{Sup}_{B(z, r)} g<g\left(u^{\prime}\right), \quad \text { so }\|g\|_{K}>0 .
$$

Let $\lambda>0$ be such that $\|\lambda g\|_{K}=1$. Then for every $k \in D$ we have:

$$
\lambda g(k) \leq \lambda g\left(u^{\prime}\right)=\varepsilon \lambda g(u) / 4 \leq \varepsilon / 4,
$$

and by symmetry of $D$, we have $\|\lambda g\|_{D} \leq \varepsilon / 4$. 
Phelps' lemma implies then:

$$
\left\|\frac{f}{\|f\|_{K}}+\lambda g\right\|_{K} \leq \varepsilon / 2 \text { or }\left\|\frac{f}{\|f\|_{K}}-\lambda g\right\|_{K} \leq \varepsilon / 2 .
$$

(Phelps' lemma is applied to the space $\left(\operatorname{Sp} K, j_{K}\right)$ : the linear space generated by $K$ equipped with the gauge (or the Minkowski functional) of $K$.)

But $f(u) /\|f\|_{K} \geq f(u) \geq 1-\varepsilon / 2>\varepsilon / 2$ (if $\left.\varepsilon \ll 1\right)$ and $\lambda g(u) \geq 0$, so we have necessarily $\|f /\| f\left\|_{K}-\lambda g\right\|_{K} \leq \varepsilon / 2$.

Then

$$
\|f-\lambda g\|_{K} \leq \frac{\varepsilon}{2}+\left\|\frac{f}{\|f\|_{K}}-f\right\|_{K} \leq \varepsilon .
$$

(ii) $\Rightarrow$ (i). (Our proof is simpler than the one given by Whitfield and Zizler [9].) Let $K$ be a compact convex subset of $X$ not containing 0 . By (ii) and the Hahn-Banach theorem there exists $g \in \operatorname{Ext}\left(B\left(X^{*}\right)\right)$ such that $\inf _{K} g>0$.

Let us first note the following easy fact:

On bounded subsets of $X^{*}$, the $w^{*}$-topology coincides with the topology $\mathscr{T}$ of uniform convergence on compact sets of $X$.

From the extremality of $g$, we deduce that there exists an $x \in S(X)$, $\delta>0$, such that:

$$
g \in S\left(B\left(X^{*}\right) ; x, \delta\right) \text { and } \operatorname{diam}_{\|\cdot\|_{K}}\left[S\left(B\left(X^{*}\right) ; x, \delta\right)\right] \leq \varepsilon,
$$

where $\varepsilon$ is defined by $3 \varepsilon=\inf _{K} g$.

Let us consider now the increasing family of balls (for $r>1$ ): $D_{r}=$ $B(r \varepsilon \mathcal{X},(r-1) \varepsilon)$, and let us show that $K \subset \dot{D}_{r}$ for some $r$.

If not, let $y \in\left[\bigcap_{r>0}\left(K \backslash \dot{D}_{r}\right)\right]$, and let $g_{r} \in S\left(X^{*}\right)$ be such that $g_{r}(r \varepsilon x-y)=\|r \varepsilon x-y\| \geq(r-1) \varepsilon$. Then $g_{r}(x) \underset{r \rightarrow \infty}{\rightarrow} 1$, and

$$
\begin{aligned}
\left(g-g_{r}\right)(y) & =g(y)+g_{r}(r \varepsilon x-y)-\varepsilon r g_{r}(x) \\
& \geq 3 \varepsilon+(r-1) \varepsilon-\varepsilon r g_{r}(x) \\
& =2 \varepsilon+r \varepsilon\left(1-g_{r}(x)\right) \geq 2 \varepsilon,
\end{aligned}
$$

which is a contradiction to the choice of $x$ and $\delta$.

REMARK. Let us show that property $(C I)$ is the "natural" intersection property which is associated to Gateaux-smoothness. In order to do this, we will describe the similarities between the dual characterizations of properties $(I)$ and $(C I)$. 
Recall first that $X$ has property $(I)$ if and only if the set of $w^{*}$-denting points of $B\left(X^{*}\right)$ is norm dense in $S\left(X^{*}\right)$ [3]. And observe that the definition of $w^{*}$-denting points (resp. extreme points) is obtained from the one of $w^{*}$-strongly exposed points (resp. $w^{*}$-exposed points) by allowing the $w^{*}$-slices not to be parallel.

2. A "Transfer Theorem" for property $(C I)$. In this section we will prove a "transfer theorem" which is analogous to the corresponding one for property $(I)$ [2]. For other "transfer theorems" see [4], [5].

In this paper all the linear operators we consider are assumed to be bounded.

Theorem 2. Let $T: X \rightarrow Y$ be a linear operator such that $T$ and $T^{*}$ are injective.

If $Y$ has an equivalent norm with property $(C I)$, then $X$ has an equivalent norm with property $(C I)$.

Proof. Recall that we denote by $\mathscr{T}\left(=\mathscr{T}_{X}\right)$ the topology on $X^{*}$ of uniform convergence on compact sets of $X$.

We decompose the proof into three steps:

1. If $T: X \rightarrow Y$ is a linear operator, then $T^{*}: Y^{*} \rightarrow X^{*}$ is $\mathscr{T}_{Y}-\mathscr{T}_{X}$ continuous.

Indeed, let $\varepsilon>0$ and let $K$ be a compact subset of $X$. Then $T(K)$ is a compact subset of $Y$, and:

$$
T^{*}\left(\left\{y^{*} \in Y^{*}: \sup _{T(K)} y^{*}<\varepsilon\right\}\right) \subset\left\{x^{*} \in X^{*}: \operatorname{Sup}_{K} x^{*}<\varepsilon\right\} .
$$

2. $X$ is the dual of $\left(X^{*}, \mathscr{T}\right)$.

Indeed, every $x \in X$ is $w^{*}$-continuous on $X^{*}$, hence $\mathscr{T}$-continuous. On the other hand, if $\xi \in\left(X^{*}, \mathscr{T}\right)^{*}$, then $\xi$ is continuous on $\left(B\left(X^{*}\right), \mathscr{T}\right)$ $=\left(B\left(X^{*}\right), w^{*}\right)$, so $\xi \in X$. (Another way to see this is to observe that $\mathscr{T}$ is coarser than the Mackey topology associated to $w^{*}$.)

It is now easy to deduce the following:

Claim. If $H$ is a subspace of $X^{*}$ which is $w^{*}$-dense in $X^{*}$, then $H$ is $\mathscr{T}$-dense in $X^{*}$.

3. If $T: X \rightarrow Y$ is such that $T^{*}$ is injective, then $X$ has an equivalent norm for which $T^{*}\left(\operatorname{Ext}\left(Y^{*}\right)\right) \subset \operatorname{Ext}\left(X^{*}\right)$.

Indeed, let $\|\cdot\|$ be the original norm of $X$, and $C=T^{*}\left(B\left(Y^{*}\right)\right)$.

Define on $X^{*}$ a convex $w^{*}$-lower-semicontinuous function by:

$$
\psi\left(x^{*}\right)=\left\|x^{*}\right\|^{*}+\int_{0}^{\infty} e^{-t} \operatorname{dist}\left(x^{*}, t C\right) d t
$$


and define the new norm on $X$ by:

$$
B_{|\cdot|^{*}}\left(x^{*}\right)=\left\{x^{*}: \psi\left(x^{*}\right) \leq 1\right\} .
$$

Remarks. (i) To see that $\psi$ is $w^{*}$-lower semicontinuous $\left(w^{*}-1\right.$. s.c.) it suffices to observe the easy (and well known) fact that for a $w^{*}$ compact subset $K$ of $X^{*}$ the functon $x^{*} \rightarrow \operatorname{dist}\left(x^{*}, K\right)$ is $w^{*}$-1.s.c.

(ii) The functional $\psi\left(x^{*}\right)$ is symmetric, i.e.: $\psi\left(x^{*}\right)=\psi\left(-x^{*}\right)$, since $C$ is, and satisfies $\left\|x^{*}\right\| \leq \psi\left(x^{*}\right) \leq 2\left\|x^{*}\right\|$; hence the set $\left\{\psi\left(x^{*}\right) \leq 1\right\}$ is the unit ball of a dual equivalent norm on $X^{*}$, which is simply the gauge of the set $\left\{\psi\left(x^{*}\right) \leq 1\right\}$.

Let $y_{0}^{*} \in \operatorname{Ext}\left(Y^{*}\right)$, and choose $t_{0}>0$ such that $\left|t_{0} T^{*}\left(y_{0}^{*}\right)\right|^{*}=1$. We want to prove that $t_{0} T^{*}\left(y_{0}^{*}\right)=x_{0}^{*} \in \operatorname{Ext}\left(B_{|\cdot| *}\left(X^{*}\right)\right)$.

Let $x_{1}^{*}, x_{2}^{*}$ be such that $2 x_{0}^{*}=x_{1}^{*}+x_{2}^{*},\left|x_{1}^{*}\right|^{*}=\left|x_{2}^{*}\right|^{*}=1$. Then $\psi\left(x_{0}^{*}\right)=\psi\left(x_{1}^{*}\right)=\psi\left(x_{2}^{*}\right)=1$, and by a convexity argument, and the fact that $t \rightarrow \operatorname{dist}\left(x^{*}, t C\right)$ is continuous for every $x^{*} \in X^{*}$, we deduce that for every $t$, we have $2 \operatorname{dist}\left(x_{0}^{*}, t C\right)=\operatorname{dist}\left(x_{1}^{*}, t C\right)+\operatorname{dist}\left(x_{2}^{*}, t C\right)$.

So $\operatorname{dist}\left(x_{1}^{*}, t_{0} C\right)=\operatorname{dist}\left(x_{2}^{*}, t_{0} C\right)=0$, but $C$ is norm closed, then $x_{1}^{*} \in t_{0} C$ and $x_{2}^{*} \in t_{0} C$.

By injectivity of $T^{*}$, and extremality of $y_{0}^{*}$, we deduce that $x_{0}^{*}$ is extremal.

The theorem is now an easy consequence of the above three facts. Indeed, give $X$ and $Y$ equivalent norms for which $\operatorname{Ext}\left(Y^{*}\right)$ is $\mathscr{T}$-dense in $Y^{*}$, and $T^{*}\left(\operatorname{Ext}\left(Y^{*}\right)\right) \subset \operatorname{Ext}\left(X^{*}\right)$. Then $T^{*}\left(\operatorname{Ext}\left(Y^{*}\right)\right)$ is $\mathscr{T}$-dense in $T^{*}\left(Y^{*}\right)$ which is itself $\mathscr{T}$-dense in $X^{*}$. The conclusion follows.

Remarks. (i). Property $(C I)$ is hereditary (a subspace of a space with an equivalent $(C I)$-norm, has an equivalent $(C I)$-norm) if and only if the above "transfer theorem" is valid without the hypothesis " $T$ * injective".

The if part is trivial.

Suppose $(C I)$ is hereditary. Let $T: X \rightarrow Y$ be an injective operator. If we factorize $T$ by its image:

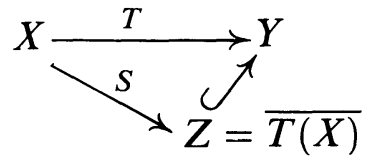

the heredity of property $(C I)$, and Theorem 2, implies that $X$ has an equivalent $(C I)$-norm if $Y$ does.

The same remark applies to Deville's "transfer theorem" for Property $(I)$ : Let $T: X \rightarrow Y$ be such that $T^{*}$ and $T^{* *}$ are injective; then $X$ has an equivalent $(I)$-norm if $Y$ does. 
(ii) It was proved in [3], that if the norm of $X$ is locally uniformly convex, then its dual norm on $X^{*}$ satisfies property $(* I)$ : every $w^{*}$-compact set is an intersection of balls.

In particular spaces $l^{\infty}(\Gamma)$ have equivalent $(C I)$-norms. Then, if property $(C I)$ is hereditary, every Banach space will have an equivalent $(C I)$-norm (since the spaces $l^{1}(\Gamma)$ have equivalent l.u.c. norms, and every Banach space is a subspace of some $l^{\infty}(\Gamma)$-space).

3. Applications. In [9], Whitfield and Zizler proved that every Banach space with a transfinite Schauder basis has an equivalent norm with property $(C I)$.

In [2], Deville uses his "transfer theorem" for property $(I)$ to prove that the James' spaces $J(\eta)$ have equivalent norms with property $(I)$.

We give here a "unified" proof of these results which is simpler than Whitfield-Zizler's proof, and give a generalization of Deville's result on $J(\eta)$ spaces.

Recall first that a family of projections $\left(P_{\alpha}\right)_{0 \leq \alpha \leq \mu}, \mu$ ordinal, is a transfinite Schauder decomposition of the Banach space $X$ if:

(i) $P_{0}=0, P_{\mu}=\mathrm{id}_{X}$

(ii) $P_{\alpha} P_{\beta}=P_{\min (\alpha, \beta)}$ for every $\alpha, \beta \leq \mu$

(iii) $\Phi:[0, \mu] \times X \rightarrow X: \Phi(\alpha, x)=P_{\alpha} x$ is separately continuous.

Such a decomposition is said to be shrinking if

$$
X^{*}=\overline{s p} \bigcup_{\alpha<\mu}\left(P_{\alpha+1}^{*}-P_{\alpha}^{*}\right)\left(X^{*}\right)
$$

The following theorem should be compared with Zizler's theorem on l.u.c. renormings [10].

THEOREM 3. Let $\left(P_{\alpha}\right)_{0 \leq \alpha \leq \mu}$ be a Schauder decomposition [resp. a shrinking Schauder decomposition] of the Banach space $X$. Suppose that for every $\alpha, 0 \leq \alpha<\mu$, the space $X_{\alpha}=\left(P_{\alpha+1}-P_{\alpha}\right)(X)$ has an equivalent norm with property $(C I)$ [resp. with property $(I)]$. Then the space $X$ has an equivalent norm with property $(C I)$ [resp. with property $(I)]$.

"Transfer theorems" for properties $(I)$ and $(C I)$ permit the proof of the theorem to be reduced to the following special case:

Proposition 4. Let $\left(X_{\alpha},\|\cdot\|_{\alpha}\right)_{\alpha \in \Gamma}$ be a family of spaces with property (CI) [resp. with property $(I)]$, then the space $X=\left(\bigoplus_{\alpha \in \Gamma} X_{\alpha}\right)_{c_{0}}$ has an equivalent norm with property $(C I)$ [resp. with property $(I)]$. 
Proof. Let $\|\cdot\|$ be an equivalent lattice norm on $c_{0}(\Gamma)$ which is $C^{\infty}$ [1]. (Lattice norms on $c_{0}(\Gamma)$ are norms satisfying the following property: If two elements $x=\left(x_{\alpha}\right)_{\alpha \in \Gamma}$, and $y=\left(y_{\alpha}\right)_{\alpha \in \Gamma}$ are such that $\left|x_{\alpha}\right| \leq\left|y_{\alpha}\right|$ for every $\alpha \in \Gamma$, then $\|x\| \leq\|y\| . C^{\infty}$ stands for infinitely Fréchet-differentiable.)

Define on $X$ an equivalent norm by:

$$
\left|\left(x_{\alpha}\right)_{\alpha \in \Gamma}\right|=\left\|\left(\left\|x_{\alpha}\right\|_{\alpha}\right)_{\alpha \in \Gamma}\right\| .
$$

A direct computation shows that its dual norm on $X^{*}=\left(\bigoplus_{\alpha \in \Gamma} X_{\alpha}^{*}\right)_{l^{1}}$ is given by $\left|\left(x_{\alpha}^{*}\right)_{\alpha \in \Gamma}\right|^{*}=\left\|\left(\left\|x_{\alpha}^{*}\right\|_{\alpha}^{*}\right)_{\alpha \in \Gamma}\right\|^{*}$.

Let $A$ be such that for every $\left(a_{\alpha}\right)_{\alpha \in \Gamma} \in c_{0}(\Gamma)$ we have

$$
\frac{1}{A} \operatorname{Sup}_{\alpha \in \Gamma}\left|a_{\alpha}\right| \leq\left\|\left(a_{\alpha}\right)_{\alpha \in \Gamma}\right\| \leq A \operatorname{Sup}_{\alpha \in \Gamma}\left|a_{\alpha}\right|
$$

First case. Property $(C I)$.

Step 1. We first show the following:

Claim. If $x^{*}=\left(x_{\alpha}^{*}\right)_{\alpha \in \Gamma} \in X^{*}$ is such that $x_{\alpha}^{*} \in \operatorname{Ext}\left(X_{\alpha}^{*}\right)$ for every $\alpha \in$ $\Gamma$, and $\left(\left\|x_{\alpha}^{*}\right\|_{\alpha}^{*}\right)_{\alpha \in \Gamma}$ is a $w^{*}$-exposed point of $l^{1}(\Gamma)$, then $x^{*} \in \operatorname{Ext}\left(X^{*}\right)$.

Proof. Let $\left(a_{\alpha}\right)_{\alpha \in \Gamma}$ be an element of $c_{0}(\Gamma)$ which exposes $\left(\left\|x_{\alpha}^{*}\right\|_{\alpha}^{*}\right)_{\alpha \in \Gamma}$ :

$$
\left\|\left(a_{\alpha}\right)_{\alpha \in \Gamma}\right\|=\left\|\left(\left\|x_{\alpha}^{*}\right\|_{\alpha}^{*}\right)_{\alpha \in \Gamma}\right\|^{*}=\sum_{\alpha \in \Gamma} a_{\alpha}\left\|x_{\alpha}^{*}\right\|_{\alpha}^{*}=1 ;
$$

then $a_{\alpha} \geq 0$ for every $\alpha \in \Gamma$.

If $2 x^{*}=x_{1}^{*}+x_{2}^{*}$, and $\left|x_{1}^{*}\right|^{*}=\left|x_{2}^{*}\right|^{*}=1$, then

$$
2=2 \sum_{\alpha \in \Gamma} a_{\alpha}\left\|x_{\alpha}^{*}\right\|_{\alpha}^{*} \leq \sum_{\alpha \in \Gamma} a_{\alpha}\left\|x_{1, \alpha}^{*}\right\|_{\alpha}^{*}+\sum_{\alpha \in \Gamma} a_{\alpha}\left\|x_{2, \alpha}^{*}\right\|_{\alpha}^{*} \leq 2 .
$$

So $\sum_{\alpha \in \Gamma} a_{\alpha}\left\|x_{1, \alpha}^{*}\right\|_{\alpha}^{*}=\sum_{\alpha \in \Gamma} a_{\alpha}\left\|x_{2, \alpha}^{*}\right\|_{\alpha}^{*}=1$.

Since $\left(a_{\alpha}\right)_{\alpha \in \Gamma}$ exposes $\left(\left\|x_{\alpha}^{*}\right\|_{\alpha}^{*}\right)_{\alpha \in \Gamma}$, we have: $\left\|x_{1, \alpha}^{*}\right\|_{\alpha}^{*}=\left\|x_{2, \alpha}^{*}\right\|_{\alpha}^{*}=$ $\left\|x_{\alpha}^{*}\right\|_{\alpha}^{*}$, for every $\alpha \in \Gamma$. And by the extremality of $x_{\alpha}^{*}$ for every $\alpha$, we have $x^{*}=x_{1}^{*}=x_{2}^{*}$.

Step 2. We will prove that the set of extreme points described in Step 1 is $\mathscr{T}$-dense in $X^{*}$.

Let $\varepsilon>0, K \subset B(X)$ be a compact subset of $X, x^{*} \in X^{*},\left|x^{*}\right|^{*}=1$. Suppose $K$ is convex and symmetric.

Put $a_{\alpha}^{*}=\left\|x_{\alpha}^{*}\right\|_{\alpha}^{*}, K_{\alpha}=\pi_{\alpha}(K)$, where $\pi_{\alpha}$ is the natural projection of $X$ onto $X_{\alpha}$. Then $K_{\alpha} \subset A B\left(X_{\alpha}\right)$. 
For each $\alpha \in \Gamma$, choose $\tilde{x}_{\alpha}^{*} \in \operatorname{Ext}\left(X_{\alpha}^{*}\right),\left\|\tilde{x}_{\alpha}^{*}\right\|_{\alpha}^{*}=1, \mu_{\alpha}^{*} \geq 0$, such that $\left\|\mu_{\alpha}^{*} \tilde{x}_{\alpha}^{*}-x_{\alpha}^{*}\right\|_{K_{\alpha}}^{*} \leq \varepsilon a_{\alpha}^{*}$.

Choose $\Gamma_{0} \subset \Gamma, \Gamma_{0}$ finite, such that $\sum_{\alpha \in \Gamma \backslash \Gamma_{0}} a_{\alpha}^{*} \leq \varepsilon$.

For $\alpha \in \Gamma_{0}$, put $\lambda_{\alpha}^{*}=\mu_{\alpha}^{*}$, and for $\alpha \in \Gamma \backslash \Gamma_{0}$, put $\lambda_{\alpha}^{*}=a_{\alpha}^{*}$. Then $\left(\lambda_{\alpha}^{*}\right)_{\alpha \in \Gamma} \in l^{1}(\Gamma)$.

Choose $\left(\tilde{\lambda}_{\alpha}^{*}\right)_{\alpha \in \Gamma}$ to be a $w^{*}$-exposed point of $l^{1}(\Gamma)$ such that:

$$
\left\|\left(\tilde{\lambda}_{\alpha}^{*}\right)_{\alpha \in \Gamma}\right\|^{*}=\left\|\left(\lambda_{\alpha}^{*}\right)_{\alpha \in \Gamma}\right\|^{*} \text { and } \sum_{\alpha \in \Gamma}\left|\tilde{\lambda}_{\alpha}^{*}-\lambda_{\alpha}^{*}\right| \leq \varepsilon
$$

By Step $1,\left(\tilde{\lambda}_{\alpha}^{*} \tilde{x}_{\alpha}^{*}\right)_{\alpha \in \Gamma}$ is an extreme point of $X^{*}$, and

$$
\begin{aligned}
& \left|\left(\tilde{\lambda}_{\alpha}^{*} \tilde{x}_{\alpha}^{*}-x_{\alpha}^{*}\right)_{\alpha \in \Gamma \mid}\right|_{K}^{*} \leq \sum_{\alpha \in \Gamma}\left\|\tilde{\lambda}_{\alpha}^{*} \tilde{x}_{\alpha}^{*}-x_{\alpha}^{*}\right\|_{K_{\alpha}}^{*} \\
& \quad \leq \sum_{\alpha \in \Gamma_{0}}\left\{A\left|\tilde{\lambda}_{\alpha}^{*}-\lambda_{\alpha}^{*}\right|+\left\|\lambda_{\alpha}^{*} \tilde{x}_{\alpha}^{*}-x_{\alpha}^{*}\right\|_{K_{\alpha}}^{*}\right\}+A \sum_{\alpha \in \Gamma \backslash \Gamma_{0}}\left\|\tilde{\lambda}_{\alpha}^{*} \tilde{x}_{\alpha}^{*}-x_{\alpha}^{*}\right\|_{\alpha}^{*} \\
& \quad \leq 2 A \varepsilon+A \sum_{\alpha \in \Gamma \backslash \Gamma_{0}}\left\{\left|\tilde{\lambda}_{\alpha}^{*}-\lambda_{\alpha}^{*}\right|+\left\|\lambda_{\alpha}^{*} \tilde{x}_{\alpha}^{*}\right\|_{\alpha}^{*}+\left\|x_{\alpha}^{*}\right\|_{\alpha}^{*}\right\} \leq 5 A \varepsilon .
\end{aligned}
$$

Second case. Property $(I)$. Recall first that a Banach space has property $(I)$ if and only if the set of $w^{*}$-denting points of $B\left(X^{*}\right)$ is norm dense in $S\left(X^{*}\right)$ [3].

Step 1. We will show the following:

Claim. If $x^{*}=\left(x_{\alpha}^{*}\right)_{\alpha \in \Gamma} \in X^{*}$ is such that $x_{\alpha}^{*} \in w^{*}$-dent $\left(X_{\alpha}^{*}\right)$ for every $\alpha \in \Gamma$, and $\left(\left\|x_{\alpha}^{*}\right\|_{\alpha}^{*}\right)_{\alpha \in \Gamma}$ is a $w^{*}$-strongly exposed point of $l^{1}(\Gamma)$, then $x^{*} \in w^{*}$-dent $\left(X^{*}\right)$.

Proof. Put $a_{\alpha}^{*}=\left\|x_{\alpha}^{*}\right\|_{\alpha}^{*}$, and let $\left(a_{\alpha}\right)_{\alpha \in \Gamma}$ be such that $\left\|\left(a_{\alpha}\right)_{\alpha \in \Gamma}\right\|=$ $\left\|\left(a_{\alpha}^{*}\right)_{\alpha \in \Gamma}\right\|^{*}=\sum_{\alpha \in \Gamma} a_{\alpha} a_{\alpha}^{*}=1$; then $a_{\alpha} \geq 0$ for every $\alpha$.

Let $\varepsilon>0$, and choose $\Gamma_{0} \subset \Gamma, \Gamma_{0}$ finite such that $\sum_{\alpha \in \Gamma \backslash \Gamma_{0}} a_{\alpha}^{*} \leq \varepsilon$ and $\inf _{\Gamma_{0}} a_{\alpha}^{*}=\delta>0$.

Choose $\eta_{1}>0$, and $x_{\alpha} \in X_{\alpha}$, for every $\alpha \in \Gamma_{0}$, such that $\left\|x_{\alpha}\right\|_{\alpha}=1$, and

$$
\left.\begin{array}{l}
x_{\alpha}\left(y_{\alpha}^{*}\right) \geq a_{\alpha}^{*}\left(1-\eta_{1}\right) \\
\left\|y_{\alpha}^{*}\right\|_{\alpha}^{*} \leq a_{\alpha}^{*}
\end{array}\right\} \Rightarrow\left\|y_{\alpha}^{*}-x_{\alpha}^{*}\right\|_{\alpha}^{*} \leq \varepsilon a_{\alpha}^{*} .
$$

For $\alpha \in \Gamma \backslash \Gamma_{0}$, pick any $x_{\alpha} \in X_{\alpha},\left\|x_{\alpha}\right\|_{\alpha}=1$.

Choose $\varepsilon^{\prime} \leq \varepsilon$, such that $1-\eta_{1} \leq\left(1-\varepsilon^{\prime} / \delta\right) /\left(1+\varepsilon^{\prime} / \delta\right)$, and let $\eta_{2}>0$ be such that

$$
\left.\begin{array}{l}
\sum_{\alpha \in \Gamma} a_{\alpha} b_{\alpha}^{*} \geq 1-\eta_{2} \\
\left\|\left(b_{\alpha}^{*}\right)_{\alpha \in \Gamma}\right\|^{*} \leq 1
\end{array}\right\} \Rightarrow \sum_{\alpha \in \Gamma}\left|b_{\alpha}^{*}-a_{\alpha}^{*}\right| \leq \varepsilon^{\prime}
$$


Now if $y^{*}=\left(y_{\alpha}^{*}\right)_{\alpha \in \Gamma}$ is such that:

$$
\sum_{\alpha \in \Gamma} a_{\alpha} x_{\alpha}\left(y_{\alpha}^{*}\right) \geq 1-\eta_{2} \text { and }\left|y^{*}\right|^{*}=\left\|\left(\left\|y_{\alpha}^{*}\right\|_{\alpha}^{*}\right)_{\alpha \in \Gamma}\right\|^{*} \leq 1,
$$

then

$$
\sum a_{\alpha}\left\|y_{\alpha}^{*}\right\|_{\alpha}^{*} \geq 1-\eta_{2} \text { and }\left\|\left(x_{\alpha}\left(y_{\alpha}^{*}\right)\right)_{\alpha \in \Gamma}\right\|^{*} \leq 1 .
$$

So we have

$$
\sum_{\alpha \in \Gamma}\left|a_{\alpha}^{*}-\left\|y_{\alpha}^{*}\right\|_{\alpha}^{*}\right| \leq \varepsilon^{\prime} \quad \text { and } \quad \sum_{\alpha \in \Gamma}\left|a_{\alpha}^{*}-x_{\alpha}\left(y_{\alpha}^{*}\right)\right| \leq \varepsilon^{\prime} .
$$

For $\alpha \in \Gamma_{0}$, we have:

$$
x_{\alpha}\left(\frac{y_{\alpha}^{*}}{\left\|y_{\alpha}^{*}\right\|_{\alpha}^{*}}\right) \geq \frac{a_{\alpha}^{*}-\varepsilon^{\prime}}{a_{\alpha}^{*}+\varepsilon^{\prime}} \geq \frac{1-\varepsilon^{\prime} / \delta}{1+\varepsilon^{\prime} / \delta} \geq 1-\eta_{1}
$$

from this we deduce $\left\|y_{\alpha}^{*}-x_{\alpha}^{*}\right\|_{\alpha}^{*} \leq \varepsilon a_{\alpha}^{*}+\left|a_{\alpha}^{*}-\left\|y_{\alpha}^{*}\right\|_{\alpha}^{*}\right|$.

Then

$$
\begin{aligned}
& \sum_{\alpha \in \Gamma}\left\|y_{\alpha}^{*}-x_{\alpha}^{*}\right\|_{\alpha}^{*} \\
& \quad \leq \sum_{\alpha \in \Gamma_{0}}\left\{\varepsilon a_{\alpha}^{*}+\left|a_{\alpha}^{*}-\left\|y_{\alpha}^{*}\right\|_{\alpha}^{*}\right|\right\}+\sum_{\alpha \in \Gamma \backslash \Gamma_{0}}\left\{\left\|x_{\alpha}^{*}\right\|_{\alpha}^{*}+\left\|y_{\alpha}^{*}\right\|_{\alpha}^{*}\right\} \\
& \quad \leq A \varepsilon+\varepsilon+\varepsilon+\sum_{\alpha \in \Gamma \backslash \Gamma_{0}}\left\{\left|\left\|y_{\alpha}^{*}\right\|_{\alpha}^{*}-a_{\alpha}^{*}\right|+a_{\alpha}^{*}\right\} \leq(A+4) \varepsilon
\end{aligned}
$$

which concludes the proof of $x^{*} \in w^{*}$-dent $\left(X^{*}\right)$.

Step 2. We will show that the set of $w^{*}$-denting points described in Step 1 is norm dense in $X^{*}$.

Let $\varepsilon>0$, and $x^{*}=\left(x_{\alpha}^{*}\right)_{\alpha \in \Gamma} \in X^{*},\left|x^{*}\right|^{*}=1$. Put $a_{\alpha}^{*}=\left\|x_{\alpha}^{*}\right\|_{\alpha}^{*}$.

For every $\alpha \in \Gamma$, choose $\tilde{x}_{\alpha}^{*} \in w^{*}-\operatorname{dent}\left(X_{\alpha}^{*}\right)$ such that $\left\|\tilde{x}_{\alpha}^{*}\right\|_{\alpha}^{*}=1$ and $\left\|a_{\alpha}^{*} \tilde{x}_{\alpha}^{*}-x_{\alpha}^{*}\right\|_{\alpha}^{*} \leq \varepsilon a_{\alpha}^{*}$.

Choose a $w^{*}$-strongly exposed point $\left(\tilde{a}_{\alpha}^{*}\right)_{\alpha \in \Gamma}$ of $l^{1}(\Gamma)$ such that $\left\|\left(\tilde{a}_{\alpha}^{*}\right)_{\alpha \in \Gamma}\right\|^{*}=1$ and $\sum_{\alpha \in \Gamma}\left|a_{\alpha}^{*}-\tilde{a}_{\alpha}^{*}\right| \leq \varepsilon$. We can suppose $\tilde{a}_{\alpha}^{*} \geq 0$ for every $\alpha$.

Then $\tilde{x}^{*}=\left(a_{\alpha}^{*} \tilde{x}_{\alpha}^{*}\right)_{\alpha \in \Gamma}$ is a $w^{*}$-denting point of $X^{*},\left|\tilde{x}^{*}\right|^{*}=1$, and

$$
\sum_{\alpha \in \Gamma}\left\|\tilde{a}_{\alpha}^{*} \tilde{x}_{\alpha}^{*}-x_{\alpha}^{*}\right\|_{\alpha}^{*} \leq \sum_{\alpha \in \Gamma}\left|\tilde{a}_{\alpha}^{*}-a_{\alpha}^{*}\right|+\left\|a_{\alpha}^{*} \tilde{x}_{\alpha}^{*}-x_{\alpha}^{*}\right\|_{\alpha}^{*} \leq(A+1) \varepsilon .
$$

This achieves the proof of Proposition 4.

Proof of Theorem 3. For every $\alpha, 0 \leq \alpha<\mu$, denote by $\pi_{\alpha}$ the operator $\left(P_{\alpha+1}-P_{\alpha}\right)$ when considered as an operator from $X$ into $\left(P_{\alpha+1}-P_{\alpha}\right)(X)=X_{\alpha}$. 
Standard argument shows that for every $x \in X$

$$
\left(\left\|P_{\alpha+1} x-P_{\alpha} x\right\|\right)_{0 \leq \alpha<\mu} \in c_{0}([0, \mu[) .
$$

Let $\|\cdot\|_{\alpha}$ be an equivalent norm on $X_{\alpha}$ with property $(C I)$ [resp. with property $(I)]$. We can suppose $\|\cdot\|_{\alpha} \leq\|\cdot\|$ on $X_{\alpha}$, for each $\alpha$, where $\|\cdot\|$ is the norm induced by $X$ on $X_{\alpha}$.

Let

$$
T: X \rightarrow Y=\left[\bigoplus_{0 \leq \alpha<\mu}\left(X_{\alpha},\|\cdot\|_{\alpha}\right)\right]_{c_{0}}: T x=\left(\pi_{\alpha}(x)\right)_{0 \leq \alpha<\mu}
$$

Then $T$ is continuous and injective.

The operator $T^{*}: Y^{*} \rightarrow X^{*}$ is given by

$$
T^{*}\left(\left(x_{\alpha}^{*}\right)_{0 \leq \alpha<\mu}\right)=\sum_{0 \leq \alpha<\mu} \pi_{\alpha}^{*}\left(x_{\alpha}^{*}\right) .
$$

Then $T^{*}$ is injective.

Moreover, $T^{*}\left(Y^{*}\right)$ is norm dense in $X^{*}$ when the decomposition is shrinking [since $\pi_{\alpha}^{*}\left(X_{\alpha}^{*}\right)=\left(P_{\alpha+1}^{*}-P_{\alpha}^{*}\right)\left(X^{*}\right)$ ].

The theorem follows in case of property $(C I)$ by our "transfer theorem", and in case of property $(I)$ by Deville's "transfer theorem" [2].

Using techniques of [8], it can be proved.

Proposition 5. Let $X$ be a Banach space with a transfinite Schauder basis, and $Y$ a space with an equivalent norm with property $(C I)$. Then the space $X \hat{\otimes}_{\rho} Y$ has an equivalent norm with property $(C I)$, for every tensor norm $\rho$.

The idea of the proof is to show that if $\left(P_{\alpha}\right)_{0 \leq \alpha \leq \mu}$ is a Schauder basis of $X$, then the family $\left(P_{\alpha} \otimes \operatorname{Id}_{Y}\right)_{0 \leq \alpha \leq \mu}$ is a Schauder decomposition of $X \hat{\otimes}_{\rho} Y$, and to apply Theorem 3 .

REMARK. If $\left(X_{n}\right)_{n \geq 1}$ is a sequence of Banach spaces with equivalent $(C I)$-norms, then $\left(\bigoplus_{n=1}^{\infty} X_{n}\right)_{l_{\infty}}$ has an equivalent $(C I)$-norm. Indeed, consider the operator $T:\left(\bigoplus_{n=1}^{\infty} X_{n}\right)_{l \infty} \rightarrow\left(\bigoplus_{n=1}^{\infty} X_{n}\right)_{c_{0}}: T\left(\left(x_{n}\right)_{n \geq 1}\right)=$ $\left(x_{n} / n\right)_{n \geq 1}$, and apply Theorem 2 .

It is not clear whether the family of spaces with equivalent $(C I)$ norms is stable under (uncountable) $l^{\infty}$-sums. 
Acknowledgment. I want to thank Robert Deville for several helpful discussions concerning this work.

\section{REFERENCES}

[1] R. Bonic and J. Frampton, Smooth functions on Banach manifolds, J. Math. Mech., 15 (1966), 877-898.

[2] R. Deville, Théorème de transfert pour la propriété des boules, to appear.

[3] J. R. Gilles, D. A. Gregory and B. Sims, Characterization of normed linear spaces with Mazur's intersection property, Bull. Aust. Math. Soc., 18 (1978), $105-123$.

[4] G. Godefroy, S. Troyansky, J. H. M. Whitfield and V. Zizler, Smoothness in weakly compactly generated Banach spaces, J. Funct. Anal., 52 (1983), 344-352.

[5] Locally uniformly rotund renorming and injections into $c_{0}(T)$, Canad. Math. Bull., 27 (1984), 494-500.

[6] S. Mazur, Uber schwache Konvergenz in den Raumen $\left(L^{p}\right)$, Stud. Math., 4 (1933), 128-133.

[7] R. R. Phelps, A representation theorem for bounded convex sets, Proc. Amer. Math. Soc., 11 (1960), 976-983.

[8] A. Sersouri, Renormage de certains espaces d'opérateurs, Math. Ann., 273 (1984), 445-459.

[9] J. H. M. Whitfield and V. Zizler, Mazur's intersection property of balls for compact convex sets, to appear: Bull. Aust. Math. Soc.

[10] V. Zizler, Locally uniformly rotund renorming and decompositions of Banach spaces, Bull. Aust. Math. Soc., 29 (1984), 259-265.

Received February 12, 1987 and in revised form June 22, 1987.

U.A. N $^{\circ} 754$ AU C.N.R.S.

UNIVERSITE PARIS VI

TOUR 46, 4ÈME ETAGE

4, Place Jussieu

75252 PARIS, FRANCE 



\section{PACIFIC JOURNAL OF MATHEMATICS}

\section{EDITORS}

V. S. VARADARAJAN

(Managing Editor)

University of California

Los Angeles, CA 90024

Herbert Clemens

University of Utah

Salt Lake City, UT 84112

R. FINN

Stanford University

Stanford, CA 94305
HERMANN FLASCHKA

University of Arizona

Tucson, AZ 85721

RAMESh A. GANGOLLI

University of Washington Seattle, WA 98195

VAUGHAN F. R. JONES

University of California

Berkeley, CA 94720
ROBION KIRBY

University of California

Berkeley, CA 94720

C. C. MOORE

University of California

Berkeley, CA 94720

HAROLD STARK

University of California, San Diego

La Jolla, CA 92093

\section{ASSOCIATE EDITORS}
R. AREnS
E. F. BECKENBACH
B. H. NEUMANN
F. WOLF
K. YOSHIDA (1906-1982)

\section{SUPPORTING INSTITUTIONS}

UNIVERSITY OF ARIZONA

UNIVERSITY OF OREGON

UNIVERSITY OF BRITISH COLUMBIA UNIVERSITY OF SOUTHERN CALIFORNIA

CALIFORNIA INSTITUTE OF TECHNOLOGY

UNIVERSITY OF CALIFORNIA

STANFORD UNIVERSITY

MONTANA STATE UNIVERSITY

UNIVERSITY OF HAWAII

UNIVERSITY OF NEVADA, RENO

UNIVERSITY OF TOKYO

NEW MEXICO STATE UNIVERSITY

UNIVERSITY OF UTAH

OREGON STATE UNIVERSITY

WASHINGTON STATE UNIVERSITY

UNIVERSITY OF WASHINGTON 


\section{Pacific Journal of Mathematics}

\section{Vol. 133, No. $1 \quad$ March, 1988}

John Anderson, Finitely generated algebras and algebras of solutions to partial differential equations $\ldots \ldots \ldots \ldots \ldots \ldots \ldots \ldots \ldots \ldots \ldots \ldots \ldots$

Junichi Aramaki, On an extension of the Ikehara Tauberian theorem . . . . . 13

Giacomo Monti Bragadin, Abstract Riemannian stratifications .......... 31

Lawrence James Brenton and Richard Hill, On the Diophantine equation

$1=\sum 1 / n_{i}+1 / \prod n_{i}$ and a class of homologically trivial complex

surface singularities .................................41

C. Bruce Hughes, Controlled homotopy topological structures $\ldots . \ldots \ldots \ldots 69$

Peter Wilcox Jones and Takafumi Murai, Positive analytic capacity but zero Buffon needle probability . ...........................999

Gary M. Lieberman, Hölder continuity of the gradient at a corner for the

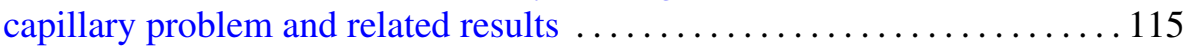

Feng Luo, Representing homology classes of $C \mathbf{P}^{2} \overline{C \mathbf{P}^{2}}$

Claudio Nebbia, Groups of isometries of a tree and the Kunze-Stein

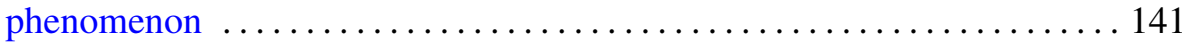

Stefan Richter, Unitary equivalence of invariant subspaces of Bergman and

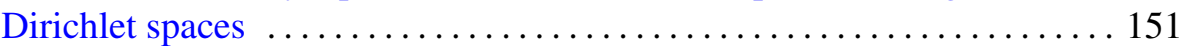

Paul Frederick Ringseth, The Selberg trace formula for groups without Eisenstein series ..................................... 157

Abderrazzak Sersouri, The Mazur property for compact sets $\ldots \ldots \ldots \ldots 185$ Alladi Sitaram, On an analogue of the Wiener Tauberian theorem for symmetric spaces of the noncompact type 